\title{
Template based matching of optical and SAR Imagery
}

\author{
Gintautas Palubinskas and Peter Reinartz \\ Remote Sensing Technology Institute \\ German Aerospace Center DLR \\ Oberpfaffenhofen \\ Gintautas.Palubinskas@dlr.de
}

\begin{abstract}
Very high resolution radar satellites such as TerraSAR-X open new possibilities for image registration due to their very accurate absolute geo-location. Thus optical and SAR imagery registration seems to be an attractive solution for accurate and automatic common tie points extraction. We propose to use template based image matching approach because it allows defining features/templates using a priori information from the scene/image. The approach works as follows: first quite complex features e.g. roundabouts/junctions are extracted in the optical image, then these features are simulated in SAR and finally they are compared/matched on patch/area basis with the SAR image. The approach is illustrated for very high resolution satellite optical WorldView-2 and radar TerraSAR-X imagery.
\end{abstract}

\section{INTRODUCTION}

Recently a great progress was achieved in orthorectification (absolute geometrical correction) of remote sensing imagery without ground control points GCPs (direct geo-referencing) e.g. such as for very high resolution optical satellite WorldView-2 data. But quite often the achieved absolute accuracy is still not sufficient for specific applications such as change detection, object recognition, scene interpretation or more generally multi-sensor data fusion. Image registration with an image of higher quality (relative geometrical correction) or known GCPs are used to enhance the orthorectification result. Both options are restricted to the availability of additional data and are very time consuming. The launch of very high resolution radar satellites such as TerraSAR-X opens new possibilities for image registration due to their very accurate absolute geolocation [1]. Thus optical and SAR imagery registration seems to be an attractive solution for accurate and automatic GCP extraction [2]. But due to different acquisition geometries of the sensors [3] this task can be quite complex especially in urban areas as already discussed e.g. in [4].

Image registration aims to align geometrically two (or more) images of the same area through identification of common features (e.g. points, lines). It consists of four main steps: feature detection, feature matching, transformation function estimation and image resampling e.g. see reviews in [5-7]. In this paper we concentrate on the first two steps which are still quite difficult especially for automatic and operational applications. Recently proposed patch based matching techniques based on mutual information e.g. [8] seem not to work robust enough.

We propose to use template based image matching approach which in general extracts features/templates in both images and then performs feature matching. Main advantage of such approach is that it allows defining features using a priori information from the scene/image. Usually point or linear features are used which are not easy to extract in SAR data robustly. Thus we propose the hybrid approach of feature based and area based matching, which first extracts more complex features/templates e.g. roundabouts in optical image, then simulates these features in SAR and finally compares/matches them on patch/area basis with SAR image. Due to quite good preliminary orthorectification of optical data the search area in SAR image can be restricted to a quite small region of interest thus reducing computation time significantly.

\section{PROCESSING CHAIN}

We propose a general processing chain for extraction of common GCPs from optical and SAR data using template based image matching (see Fig. 1) somewhat following the experience discussed in [9].

First, optical data are pan-sharpened using the panchromatic band in order to increase the resolution of multispectral data e.g. using High Pass Filter Method [10], then, data are orthorectified and atmospherically corrected using available meta-data and DEM e.g. [11, 12].

Secondly, SAR data undergo the following three preprocessing steps: orthorectification using the same DEM, despeckling e.g. [13] and finally local incidence angle correction (Digital Number (DN) to sigma naught $\sigma_{0}$ conversion e.g. [14]). Here we have to note that the DEM quality significantly influences the SAR orthoimage accuracy.

Finally, template based matching (e.g. cross-correlation of corresponding patches) leads to common GCPs which are compared to reference data if available in the last quality assessment step. Extracted GCPs can be used for various purposes e.g. optical data orthorectification enhancement, image registration/transformation and SAR data interpretation. 


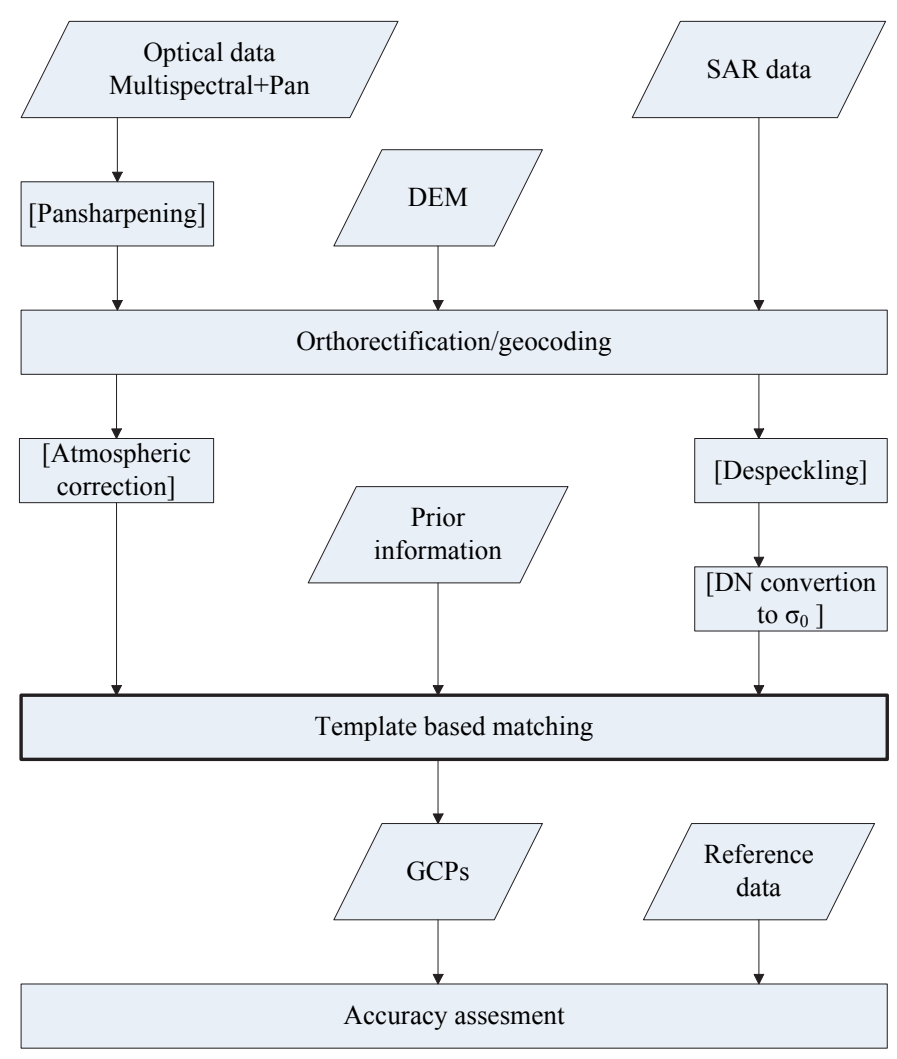

Fig. 1. General processing chain for template based matching of optical and SAR data. In brackets [] optional steps are shown.

\section{A. Template based matching}

Template based matching (see Fig 2) consists mainly of the three steps: 1) template definition and extraction in optical data (in this work template is roundabout which is defined as inner disk with streets around it), 2) template training and simulation in SAR data (see e.g. $[15,16]$ if object height information is available) and finally 3) SAR template area based matching with SAR data (cross-correlation or similarity measure CMSC [17] can be used).

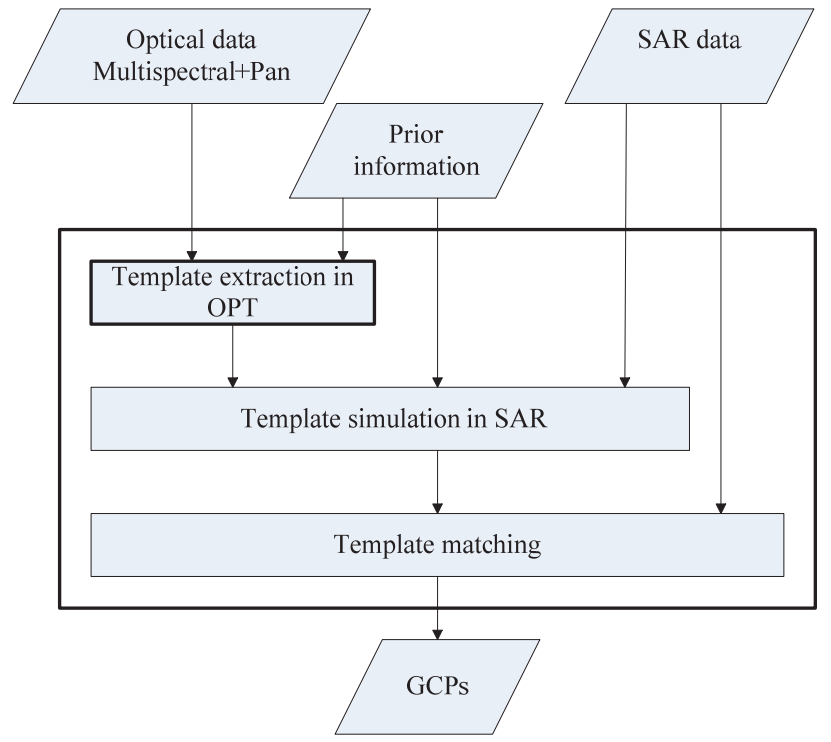

Fig. 2. Template based matching.

\section{1) Template extraction in optical data}

Template (in this case roundabout) extraction is performed in the following way. First Normalized Differenced Vegetation Index NDVI is calculated as $N D V I=(N I R-R) /(N I R+R)$, where $N I R$ and $R-$ near infrared and red bands respectively. Then NDVI image is classified into vegetation and non-vegetation classes using thresholding of the histogram. Further, the vegetation mask is labeled and morphological filtering is performed to reduce noise and small holes. Finally prior information e.g. size, radius and shape is used to extract the inner disk covered by vegetation and surrounding roads to form a roundabout template.

Here we have to note that the whole approach is not limited to a specific template. Roundabout is used this work due to its simplicity and already available positive experience from manual GCPs extraction. Other template e.g. road junction extraction will require to change this step.

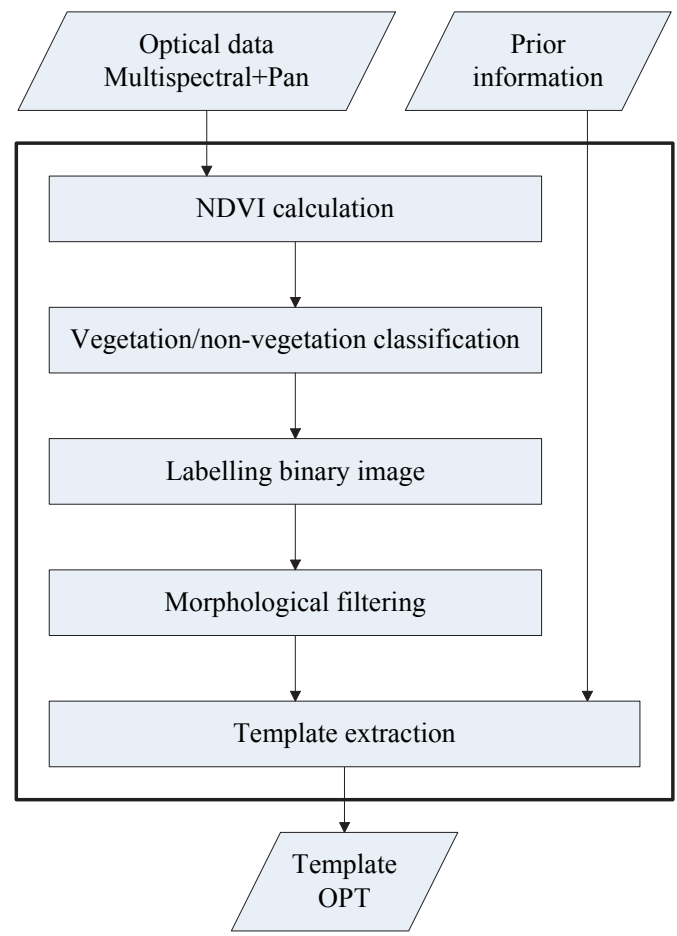

Fig. 3. Template extraction in optical data.

\section{2) Template simulation in SAR data}

First, the optical template (extracted in the previous step) is scaled to the geometry of SAR data. Knowledge of the height information can lead to more accurate simulation results $[15,16]$. Further, training of the template on SAR data is needed in order to simulate a radiometrically similar SAR template. The training can be performed for already known templates in advance by estimating statistics of vegetation and roads in absolutely calibrated SAR data. The template form can be rectangular or circular or any other form depending on the application.

\section{3) Template matching}

The SAR template is matched on patch/area basis with a region of interest (search area is limited by the preliminary 
orthorectification accuracy) of the SAR image e.g. using cross-correlation (Fig 5). The correlation peak shows the coordinates of the common GCP (Fig 6) between optical and SAR data. For matching the following similarity criteria such as Pearson's correlation, rank-correlation or any other similarity measure e.g. CMSC [17] can be used.

\section{DATA}

We shall illustrate our approach for satellite TerraSAR-X (TS-X) and WorldView-2 (WV-2) data over Oberpfaffenhofen, Germany (Table I). Multispectral data are pansharpened using High Pass Filtering Method (HPFM) [10] and are orthorectified using the SRTM DEM. Digital Orthophotos (DOP) of the Federal Agency for Cartography and Geodesy are used as reference data for validation purposes. Sizes for WV-2 multispectral (MS) images are: 2554x2666 (2010), 4774x4420 (2011) and 4190x5116 (2013) pixels.

TABLE I. SCENE PARAMETERS FOR TS-X, WV-2 AND DOP DATA

\begin{tabular}{|l|l|l|l|}
\hline \multicolumn{1}{|c|}{ Sensor } & \multicolumn{1}{|c|}{ TS-X } & \multicolumn{1}{c|}{ WV-2 } & \multicolumn{1}{c|}{ DOP } \\
Parameter & & & \\
\hline Image date & 26.11 .2011 & 10.07 .2010 & 25.6 .2012 \\
Image date & 19.07 .2014 & 22.08 .2011 & - \\
Image date & - & 04.09 .2013 & - \\
Mode & HS & PAN, MS & RGB \\
Product level & EEC & LV2A & Ortho \\
Resolution $(\mathrm{m})$ & 1.0 & $0.5,2.0$ & 0.2 \\
\hline
\end{tabular}

\section{EXPERIMENTS AND RESULTS}

In this work as a template we use roundabout (RA) which is defined as follows: inner disc usually covered by vegetation and incoming and outgoing roads (Fig. 4). In total 5 RAs were present in used WV-2 scenes, except WV-2 2010, where $2^{\text {nd }} R A$ was not yet present during acquisition time.

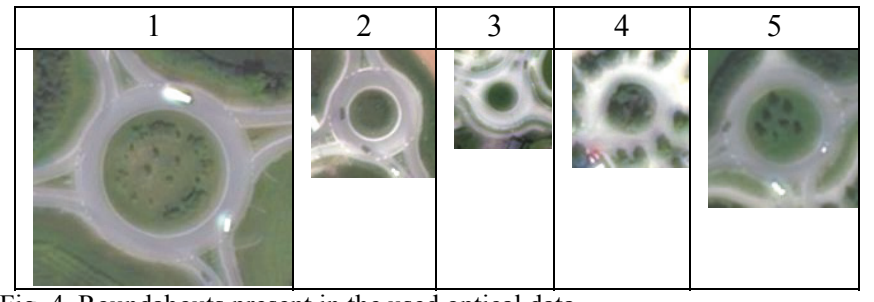

Fig. 4. Roundabouts present in the used optical data.

The SAR template extracted from optical data and trained on SAR data is correlated with a SAR image by shifting it pixel-wise in the search area of SAR image (Fig. 5). The black area in the SAR template is masked out for the matching process. The result of template matching (correlation) is illustrated in Fig. 6. The position of the correlation peak stands for a detected matching point that can be used as GCP between optical and SAR data.

The confusion matrix presented in Table II is used to assess RA detection quality for all six possible optical and SAR data combinations. The two following measures are used to characterize the quality of detection. Positive prediction value or correctness $C O R=T P /(T P+F P)$, where $T P$ - true positive and $F P$ - false positive, and true positive rate or completeness $C O M=T P /(T P+F N)$, where $F N$ - false negative.
For example, from Table II $C O R=C O M=0.9286$ and thus the achieved accuracy above $90 \%$ is very promising.

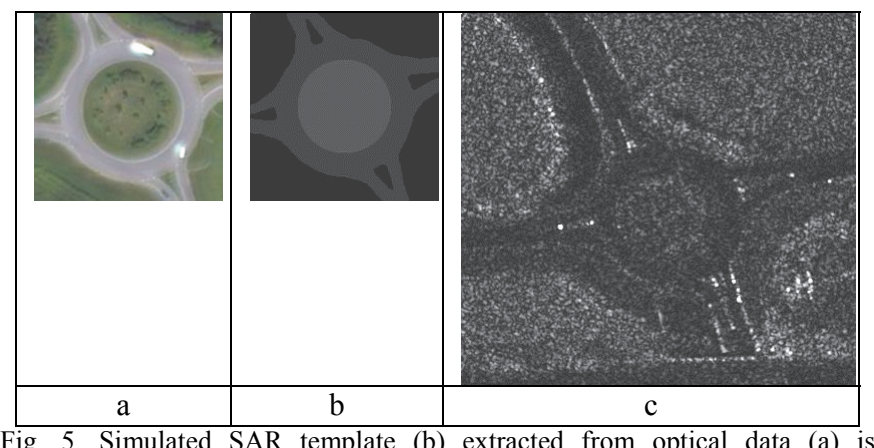

Fig. 5. Simulated SAR template (b) extracted from optical data (a) is correlated on area basis by shifting it in SAR image (c).

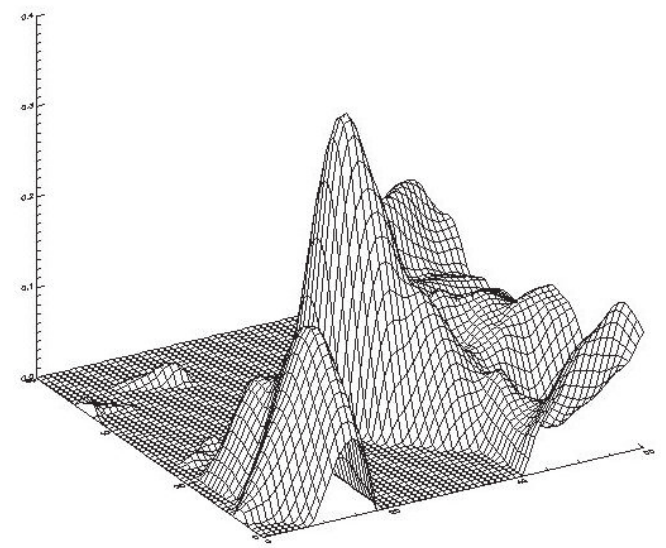

Fig. 6. Correlation peak of matching result.

TABLE II. CONFUSION MATRIX FOR TEMPLATE MATCHING IN SIX PAIRS OF WV-2 AND TS-X DATA (POSITIVE - RA, NEGATIVE - NON-RA)

\begin{tabular}{|l|l|l|l|}
\hline & \multicolumn{3}{|c|}{ Truth } \\
\hline \multirow{3}{*}{ Detection } & & positive & negative \\
\cline { 2 - 4 } & positive & $\mathrm{TP}=26$ & $\mathrm{FP}=2$ \\
\cline { 2 - 4 } & negative & $\mathrm{FN}=2$ & $\mathrm{TN}=30700$ \\
\hline
\end{tabular}

Evaluation of center positions for five RAs (four RAs in WV-2 2010, because the $2^{\text {nd }}$ RA was not yet present at the time of data acquisition) is presented in Tables III-V. We see that reference (DOP) and detected RA center position differences (RMSE) decrease from about $4 \mathrm{~m}$ to $2.5 \mathrm{~m}$ when using SAR data. If optical data are already very accurate as WV-2 2010 then no accuracy enhancement is observed. Here we have to note that optical data enhancement is limited to the SAR orthoimage accuracy (DEM quality).

\section{CONClusions}

A new template based matching approach is proposed for matching of very high resolution optical and SAR imagery. Accuracy assessment for WV-2 and TS-X data shows a great potential of the proposed method. Further research will be directed towards definition of other features: road junctions, exploitation of more prior information: OpenStreetMap and tests for different sensors: IKONOS, QuickBird and other TS$\mathrm{X}$ modes: StripMap as well as other test sites. 


\section{ACKNOWLEDGMENT}

We would like to thank DigitalGlobe and European Space Imaging (EUSI) for the collection and provision of WorldView-2 scenes. TerraSAR-X data were provided by DLR through the Science Project MTH0948.

\section{REFERENCES}

[1] M. Eineder, C. Minet, P. Steigenberger, X.Y. Cong, and T. Fritz, "Imaging geodesy - toward centimeter-level ranging accuracy with TerraSAR-X," IEEE T Geosci Remote, 49(2), 661-671, 2011.

[2] P. Reinartz, R. Müller, P. Schwind, S. Suri, and R. Bamler, "Orthorectification of VHR optical satellite data exploiting the geometric accuracy of TerraSAR-X data," ISPRS J Photogramm, 66(1), 124-132, 2011.

[3] G. Palubinskas, P. Reinartz, and R. Bamler, "Image acquisition geometry analysis for the fusion of optical and radar remote sensing data," Int Journal of Image and Data Fusion, 1(3), 271-282, 2010.

[4] J. Inglada, and A. Giros, "On the possibility of automatic multisensor image registration," IEEE T Geosci Remote, 42(10), 2104-2120, 2004.

[5] L.G. Brown, "A survey of image registration techniques," $A C M$ Computing Surveys, 24, 326-376, 1992.

[6] B. Zitova, and J. Flusser, "Image registration methods: a survey," Image and Vision Computing, 21, 977-1000, 2003.

[7] Z. Xiong, and Y. Zhang, "A critical review of image registration methods," Int Journal of Image and Data Fusion, 1(2), 137-158, 2010.
[8] S. Suri, and P. Reinartz, "Mutual information based registration of TerraSAR-X and IKONOS imagery in urban area," IEEE T Geosci Remote, 48(2), 939-949, 2010.

[9] G. Palubinskas, "How to fuse optical and radar imagery?," Proc. IGARSS, Munich, Germany, 2012.

[10] G. Palubinskas, "Fast, simple and good pan-sharpening method," Journal of Applied Remote Sensing, 7(1), 1-12, 2013.

[11] R. Müller, T. Krauß, M. Schneider, and P. Reinartz, "Automated georeferencing of optical satellite data with integrated sensor model improvement," Photogramm Eng Rem S, 78(1), 61-74, 2012.

[12] R. Richter, D. Schläpfer, and A. Müller, "An automatic atmospheric correction algorithm for visible/NIR imagery," Int J Remote Sens, vol. 27, no. 9/10, 2077-2085, 2006.

[13] C.A. Deledalle, L. Denis, and F. Tupin, "Iterative weighted Maximum Likelihood denoising with probabilistic patch-based weights," IEEE T Image Process, 18(12), 2661-2672, 2009.

[14] T. Fritz, et al., "TerraSAR-X Ground Segment Basic Product Specification Document," Issue 1.9, Report TX-GS-DD-3302, Earth Observation Center, DLR, Germany, 2013.

[15] J. Tao, G. Palubinskas, S. Auer, and P. Reinartz, "Interpretation of SAR images in urban areas using simulated optical and radar images," Proc. of JURSE, Munich, Germany, 2011.

[16] J. Tao, S. Auer, G. Palubinskas, P. Reinartz, and R. Bamler, "Automatic SAR simulation technique for object identification in complex urban scenarios," IEEE J-STARS, 7(3), 994-1003, 2014.

[17] G. Palubinskas, "Mystery behind similarity measures MSE and SSIM," Proc. of ICIP, Paris, France, 2014.

TABLE III. POSITION DIFFERENCES (IN METER) OF DETECTED ROUNDABOUTS IN OPTICAL DATA (WV-2 2010) AND REFERENCE DATA (DOP)

\begin{tabular}{|c|c|c|c|c|c|c|c|c|c|}
\hline & \multicolumn{3}{|c|}{ WV-2-2010 no matching } & \multicolumn{3}{|c|}{ WV-2 2010 matching with TS-X-2011 } & \multicolumn{3}{|c|}{ WV-2 2010 matching with TS-X-2014 } \\
\hline Roundabout & $\Delta \mathrm{x}$ & $\Delta \mathrm{y}$ & RMSE & $\Delta \mathrm{x}$ & $\Delta \mathrm{y}$ & RMSE & $\Delta \mathrm{x}$ & $\Delta y$ & RMSE \\
\hline 1 & -1.54 & 1.1 & 1.89 & -3.36 & -1.4 & 3.64 & 1.14 & 0.1 & 1.14 \\
\hline 2 & \multicolumn{9}{|c|}{ missing in optical data } \\
\hline 3 & -2.51 & 0.2 & 2.52 & 0.16 & -1.7 & 1.71 & -0.34 & -1.2 & 1.25 \\
\hline 4 & -3.04 & 0.4 & 4.39 & -2.21 & -1.4 & 2.62 & -5.21 & -0.4 & 5.23 \\
\hline 5 & \multicolumn{9}{|c|}{ not detected } \\
\hline Average & -2.36 & 0.57 & 2.49 & -1.80 & -1.5 & 2.65 & -1.47 & -0.5 & 2.54 \\
\hline
\end{tabular}

TABLE IV. POSITION DIFFERENCES (IN METER) OF DETECTED ROUNDABOUTS IN OPTICAL DATA (WV-2 2011) AND REFERENCE DATA (DOP)

\begin{tabular}{|c|c|c|c|c|c|c|c|c|c|}
\hline & \multicolumn{3}{|c|}{ WV-2-2011 no matching } & \multicolumn{3}{|c|}{ WV-2 2011 matching with TS-X-2011 } & \multicolumn{3}{|c|}{ WV-2 2011 matching with TS-X-2014 } \\
\hline Roundabout & $\Delta \mathrm{x}$ & $\Delta y$ & RMSE & $\Delta \mathrm{x}$ & $\Delta y$ & RMSE & $\Delta \mathrm{x}$ & $\Delta y$ & RMSE \\
\hline 1 & 0.97 & -3.7 & 3.83 & -2.86 & 1.6 & 3.28 & 1.14 & -0.4 & 1.21 \\
\hline 2 & 1.03 & -3.7 & 3.84 & 0.77 & -0.5 & 0.92 & 4.27 & -2.5 & 4.95 \\
\hline 3 & 0.35 & -4.6 & 4.61 & 0.16 & -1.7 & 1.71 & -0.34 & -1.2 & 1.25 \\
\hline 4 & -0.87 & -4.3 & 4.39 & -1.21 & -0.9 & 1.51 & -4.71 & -0.4 & 4.73 \\
\hline 5 & -0.60 & -4.6 & 4.64 & -2.73 & -3.2 & 4.21 & -0.23 & -1.7 & 1.72 \\
\hline Average & 0.18 & -4.18 & 4.26 & -1.17 & -0.94 & 2.32 & 0.03 & -1.24 & 2.77 \\
\hline
\end{tabular}

TABLE V. POSITION DIFFERENCES (IN METER) OF DETECTED ROUNDABOUTS IN OPTICAL DATA (WV-2 2013) AND REFERENCE DATA (DOP)

\begin{tabular}{|c|c|c|c|c|c|c|c|c|c|}
\hline & \multicolumn{3}{|c|}{ WV-2-2013 no matching } & \multicolumn{3}{|c|}{ WV-2 2013 matching with TS-X-2011 } & \multicolumn{3}{|c|}{ WV-2 2013 matching with TS-X-2014 } \\
\hline Roundabout & $\Delta \mathrm{x}$ & $\Delta \mathrm{y}$ & RMSE & $\Delta \mathrm{x}$ & $\Delta y$ & RMSE & $\Delta \mathrm{x}$ & $\Delta \mathrm{y}$ & RMSE \\
\hline 1 & -0.50 & -2.9 & 2.94 & -4.86 & -0.4 & 4.88 & 0.64 & 0.1 & 0.65 \\
\hline 2 & -0.39 & -2.5 & 2.53 & 0.27 & -1.0 & 1.04 & 3.77 & -2.5 & 4.52 \\
\hline 3 & -1.20 & -3.5 & 3.70 & -0.84 & -2.2 & 2.35 & -0.34 & -1.2 & 1.25 \\
\hline 4 & -1.89 & -3.6 & 4.07 & -1.71 & -0.9 & 1.93 & -4.71 & -0.4 & 4.73 \\
\hline 5 & -1.77 & -4.4 & 4.74 & -1.73 & -2.7 & 3.21 & 0.27 & -1.7 & 1.72 \\
\hline Average & -1.15 & -3.38 & 3.60 & -1.77 & -1.44 & 2.68 & -0.07 & -1.14 & 2.57 \\
\hline
\end{tabular}

